\title{
Human cerebral malaria and experimental cerebral malaria in mice: relevance and applicability
}

\section{Mini review}

Vectors Borne Disease (VBD)s are reported to represent amount $17 \%$ of all the infectious diseases. The geographical distribution of vectors depends upon various climatic and social factors. In the recent past, the spread of VBDs across the world is so rapid that is associated with the change in climatic factors, global warming, travel and trade, unplanned urbanization, and deforestation etc. Malaria is the leading cause of death due to VBDs and rated among the infectious diseases. According to World Health Organization (WHO), in 2015 an estimated 212 million cases of malaria occurred worldwide with 429,000 mortality, mostly children in the African Region. This review addresses the pivotal questions on the availability of animal models to develop therapeutic interventions for cerebral malaria (CM). Though, the research on experimental cerebral malaria (ECM) is published elsewhere, the applicability of such findings to human cerebral malaria (HCM) remains a myth owing to the differences in the fundamental mechanism of sequestration of parasites. Superficially, the mechanisms of parasite recognition looks cohesive between HCM and ECM, nevertheless, there are discrepancies in extrapolating the information to human. This review summarises the current research on ECM which foretells immunopathology as the main cause of CM, whereas HCM reveals the sequestration of parasitized (pRBCs) in brain.

Plasmodium falciparum infection in children and adults cause cerebral malaria $(\mathrm{CM})$ which is a primary cause of death in both the groups. Research on CM is till elusive. Both animal and human studies reveal various complicated features for development of $\mathrm{CM}$ such as increased proinflammatory cytokines, adhesion molecules, cytoadherence of parasite infected erythrocytes, platelets, WBCs in microvasculature of CNS. ${ }^{1-3}$ Plasmodium falciparum infection in human causes hearing loss in adult, ${ }^{4}$ mental health disorders in children. Past evidences in people affected with CM suggests that even after recovery, brain injury in terms of cognitive deficits and neurological deficits are noted in almost $25 \%$ of patients. ${ }^{5}$ There are subtle differences in immune pathology of Plasmodium berghei ANKA (PbA) infected mice and Plasmodium yoelii 17XL (PyXL) infected mice, where the former develops $\mathrm{CM}$ and later dies of parasitemia without neurological manifestations.

Research in human form of $\mathrm{CM}$ has posted various questions such as, is it possible to develop a model to study the human cerebral malaria by mimicking in mouse model? The answer is positive as Plasmodium berghei ANKA infection can be created in CB57BL $/ 6$ or CBA mice and it is widely used murine model to study experimental cerebral malaria (ECM). To look at the mechanisms to compare HCM and ECM, there are fundamental differences in parasite sequestration by human and mouse in CM. In mouse, the leukocytes get infected with parasites during ECM and these cells do get sequestered in brain, whereas, HCM, (which is a natural occurrence of the infection) is characterised by intense intracerebral sequestration of parasitized RBCS (pRBCs) in Brain. Extrapolation of the findings of the ECM to $\mathrm{CM}$ of human is quite complicated as mice lacking WBCs may not catch up the ECM. ${ }^{6}$ However, still large numbers of research

\author{
Volume 2 Issue I - 2017
}

\author{
Jayalakshmi Krishnan \\ Department of Life Sciences, Central University of Tamil Nadu, \\ India
}

Correspondence: Jayalakshmi Krishnan, Department of Life Sciences, Central University of Tamil Nadu, India, Email jayalakshmi@cutn.ac.in

Received: August 12, 2017| Published: October 16, 2017

publications are being done in ECM. Magnetic Resonance Imaging (MRI) studies in ECM reveal that Blood Brain Barrier (BBB) permeability was high in areas such as lateral ventricle, olfactory bulb and brainstem followed by brain swelling and edema. This effect was due to perforin, an effector molecule of having cytolytic effect, as inhibitor studies of perforin in ECM reveal the protection from BBB leakage. ${ }^{7}$ Further, in experimental CM, mice infected with Plasmodium berghei ANKA (PbA) strain, MRI showed hippocampal abnormalities. Treatment of NMDA receptor antagonists, reversed the changes, offered neuroprotective effects in both frontal cortex and hippocampus. $^{8}$

A study demonstrated that astrocytes readily take up the parasite derived vesicles and microglia the resident immune cell of brain phagocytosize the pRBCs in ECM. During such process, microglial cells release high amount of interferon gamma inducible protein 10 (IP10) in plasma as well brain tissue of infected mice. ${ }^{9}$ This suggests that if there is any such similar mechanism could be seen in HCM. Ironically, CD8+T Cells is involved in brain pathology by inducing vascular breakage and neuronal death. ${ }^{10}$ Knockout studies reveal that $\operatorname{Irgm3} 3-$ - mice were protected from CM. This protection of Irgm3-/mice was due to less recruitment of $\mathrm{CD} 8+\mathrm{T}$ cells within the brain and low production of inflammatory cytokines. ${ }^{11}$ Further studies on immunopathological changes reported that in ECM, pRBCs can be seen in the brain on three days of infection, tissue changes and edema on five days of infection followed by haemorrhage in different areas of brain at $7^{\text {th }}$ days of infection. ${ }^{12}$ The underlying immunopathological change were shown to affect the neurological functions by compromising memory. It is interesting to note that even before BBB disruption, $\mathrm{PbA}$ infected mice showed short term memory impairment and spatial memory deficits. PbA-infection induced early short term and spatial memory defects, prior to blood brain barrier (BBB) disruption. ${ }^{13}$ This was due to IL-33 receptor ST2 causing neurological inflammation and cognitive dysfunctions. While the immuno pathology at ECM is highly investigated, the role of enzymes in accelerating or preventing ECM is still poorly understood. Some studies on this have shown that, DUB cylindormatosis (CYLD), an enzyme which acts as an inhibitor of several cellular signalling pathways, is critically involved in promoting the ECM. Knockout studies of Cyld ${ }^{-1-}$ mice have survived the infection, whereas, congenic C57BL/6 mice, shown disrupted $\mathrm{BBB}$, enhanced parasite sequestration etc. Interestingly, the sequestration of CD8 T cells, have reduced in ECM brain. ${ }^{14}$ 
ECM is investigated for immuno pathology, micrRNA regulation and enzyme studies; however, the relevance of direct applicability of such findings to HCM remains elusive. Still long way is waiting to develop an intervention based on the available data generated from ECM. To be noted, immuno pathological process play a significant role in murine ECM, however, in human $\mathrm{CM}$, it is the sequestered pRBCs in endothelium, the role of immune mechanisms are still elusive in case of human CM. Second, in research on ECM, the mice can be investigated on before malaria, during malaria and after malaria, such situations seldom is possible with human. A patient first comes with malaria to hospital, treatment with anti-malarial drugs are done followed by clinical investigations. Interventions based on murine model is questionable here as studies a controlled environment offers the expected results.

\section{Acknowledgements}

None.

\section{Conflict of interest}

The author declares no conflict of interest.

\section{References}

1. Miller LH, Good MF, Milon G. Malaria pathogenesis. Science. 1994;264:1878-1883.

2. Idro R, Jenkins NE, Newton CR. Pathogenesis, clinical features, and neurological outcome of cerebral malaria. Lancet Neurol. 2005;4(12):827-840.

3. Kwiatkowski D, Hill AV, Sambou I, et al. TNF concentration in fatal cerebral, nonfatal cerebral, and uncomplicated Plasmodium falciparum malaria. Lancet. 1990;336(8725):1201-1204

4. Tada T, Hitani A, Honda NH, et al. A case of falciparum malaria: Acute hearing loss as the initial symptom. J Infect Chemother. 2007;23(1):5658.
5. John CC, Bangirana P, Byarugaba J, et al. Cerebral malaria in children is associated with long-term cognitive impairment. Pediatrics. 2008;122:e92-99.

6. White NJ, Turner GD, Medana IM, et al. The murine cerebral malaria phenomenon. Trends Parasitol. 2010;26(1):11-15.

7. Huggins MA, Johnson HL, Jin F, et al. Perforin expression by CD8 T cells is sufficient to cause fatal brain edema during experimental cerebral malaria. Infect Immun. 2017;85(5):e00985-00116.

8. Miranda AS, Vieira LB, Lacerda-Queiroz N, et al. Increased levels of glutamate in the central nervous system are associated with behavioral symptoms in experimental malaria. Braz J Med Biol Res. 2010;43(12):1173-1177.

9. Shrivastava SK, Dalko E, Delcroix-Genete D, et al. Uptake of parasitederived vesicles by astrocytes and microglial phagocytosis of infected erythrocytes may drive neuroinflammation in cerebral malaria. Glia. 207;65(1):75-92.

10. Swanson PA, Hart GT, Russo MV, et al. CD8+ T cells induce fatal brainstem pathology during cerebral malaria via luminal antigen-specific engagement of brain vasculature. PLoS Pathog. 2016;12(12):e1006022.

11. Guo J, Mc Quillan JA, Yau B, et al. IRGM3 contributes to immunopathology and is required for differentiation of antigen-specific effector CD8 + T cells in experimental cerebral malaria. Infect Immun. 2015;83(4):1406-1417.

12. Khandare AV, Bobade D, Deval M, et al. Expression of negative immune regulatory molecules, pro-inflammatory chemokine and cytokines in immunopathology of ECM developing mice. Acta Trop. 2017;172:5863.

13. Reverchon F, Mortaud S, Sivoyon M, et al. IL-33 receptor ST2 regulates the cognitive impairments associated with experimental cerebral malaria. PLoS Pathog. 2007;13(4):e1006322.

14. Schmid U, Stenzel W, Koschel J, et al. The deubiquitinating enzyme cylindromatosis dampens CD8+ T cell responses and is a critical factor for experimental cerebral malaria and blood-brain barrier damage. Front Immunol. 2017;8:27. 